\title{
In situ Visualization of Ferrous Ions Dissolved from Pure Iron Wire into Thin Frozen Aqueous Solution Films by Combination of Microscopy and Image Processing
}

\author{
Arinori Inagawa, ${ }^{*}$ Minami Maeda, and Nobuo Uehara ${ }^{* *}$ \\ Faculty of Engineering, Utsunomiya University, 7-1-2 Yoto, Utsunomiya, Tochigi 321-8585, Japan
}

(Received May 30, 2021; accepted July 22, 2021; online published August 5, 2021)

Keywords: iron, corrosion, thin ice layer, freeze-concentrated solution, image processing

The dissolution of ferrous ions from pure iron into freeze-concentrated solutions (FCSs) formed in thin films of aqueous salt solutions was visualized by a combination of in situ diffusive-reflection microscopy and image processing. An iron surface in contact with an FCS was observed with a diffusive-reflection microscope. Ferrous ions dissolved from the iron surface were complexed with 1,10-phenanthroline doped in the ice film to develop reddish complexes. In situ observation enabled us to monitor the dissolution and migration behavior of ferrous ions in the FCS. Processing of the acquired microscopic images enabled quantitative mapping of the dissolved ferrous ions. The present approach has the potential for fast screening to assess the corrosion behavior of steel materials in contact with frozen aqueous electrolytes.

\section{Introduction}

Steels have been commonly employed to construct mechanical objects due to their strength, ease of processing, and surface modification. On the other hand, corrosion induced by chemical reactions easily occurs at the surface of steels, leading to their deterioration. ${ }^{(1-3)}$ The corrosion of iron is mainly through the deposition of water containing salts on the surface and subsequent oxidation to ferrous ions. The resulting ferrous ions are then dissolved into the contacting aqueous phase. Steel materials are widely used under various conditions, including extremely high/low temperatures, humidity levels, and salt concentrations, and these severe conditions may facilitate their deterioration. In particular, in polar environments, steel materials are often exposed to frozen aqueous solutions such as sea ice and snow. When an aqueous solution is frozen, phase separation occurs to generate pure ice crystals and highly concentrated solutions of solutes, known as freeze-concentrated solutions (FCSs). ${ }^{(4)}$ In FCSs, some unusual chemical phenomena including the acceleration of chemical reactions under subzero conditions and dissolution of chemical species insoluble in water have been reported. ${ }^{(5-12)}$ These effects are attributed to the unique physicochemical properties of FCSs, induced by the microscale phase transitions at ice/solution interfaces. ${ }^{(13-15)}$ The reductive reactions of metal oxides and their dissolution have also been reported to be accelerated in FCSs. ${ }^{(16-18)}$ These results suggest that a

*Corresponding author: e-mail: ainagawa@cc.utsunomiya-u.ac.jp

** Corresponding author: e-mail: ueharan@cc.utsunomiya-u.ac.jp

https://doi.org/10.18494/SAM.2022.3448

ISSN 0914-4935 (C) MYU K.K. https://myukk.org/ 
frozen system deteriorates the properties and quality of metallic materials in contact with the system. Thus, it is essential to elucidate the corrosion behavior under such conditions to prevent the unintended corrosion of steel materials therein. For steel materials, pioneering works revealed that the oxidation of iron by dissolved halide ions and oxygen is the dominant mechanism of corrosion, resulting in the dissolution of ferrous ions at steel surfaces and their dissolution into aqueous solutions. ${ }^{(19)}$ Thus, dissolved ferrous ions are potentially a valuable tracer for corrosion, and a direct measurement system for the ions can be a powerful tool to determine corrosion spots and evaluate the corrosion rate.

The most common approach to investigating the chemical reactions in an FCS in contact with a metal surface is to freeze mixtures of metals and aqueous solutions in test tubes, then thaw the resultant solutions for chemical analysis. ${ }^{(17,18)}$ However, such a methodology can interrupt the fine time-resolved elution behavior. In addition, the contact area between FCSs and metal materials is unpredictable due to stochastic aspects of freezing phenomena, leading to less precise analysis of reaction rates when estimating reaction models. Electrochemical approaches including voltammetry are also attractive strategies for monitoring the chemical reactions in FCSs. Okada and coworkers reported the dissolution of ferrous ions from iron oxide ${ }^{(20)}$ and viologens, ${ }^{(21)}$ elucidated by cyclic voltammetry. Although electrochemical approaches can elucidate kinetic and thermodynamic parameters, it is still challenging to control the area of the surface in contact with an FCS and to obtain distribution maps of the chemical species therein when working electrodes are used. Some researchers have employed fluorescence microscopes to monitor the chemical reactions in an FCS to overcome these issues. ${ }^{(10,22,23)}$ Although numerous fluorescent probes to detect various types of metal ions have been developed, those for ferrous ions have intrinsic issues including redox reactions to generate organic fluorescent probes. ${ }^{(24)}$ Excitation by strong light such as a laser may decompose a probe. Since an FCS is confined by ice crystals, molecular diffusion is spatially limited. Thus, decomposed probes are hardly compensated by diffusion in the FCS, which is unfavorable for time course measurement by laser-induced fluorescence microscopy. The utilization of a fluorescence quenching reaction could overcome this problem. Thionine ${ }^{(25)}$ and calcein blue ${ }^{(26)}$ have been reported to be photochemically quenched with ferrous ions by an electron transfer reaction. However, the results show poor agreement with Stern-Volmer plots, indicating that the quantification cannot be easily adopted. Thus, the fluorescence approach to detecting ferrous ions requires further investigation.

In this paper, we report a diffusive-reflection-microscopy-based evaluation method to study the dissolution of the ferrous surface of iron from pure iron materials into FCSs formed in thin films of frozen aqueous solutions. The advantage of diffusive-reflection microscopy is that reflection- or absorption-based colorization can be adopted. ${ }^{(27,28)}$ The utilization of a thin-film ice sample can reduce the area of contact between the FCS and the iron materials, enabling quantitative evaluation of the iron dissolution. The dissolved ferrous ions were detected by their complexation with 1,10-phenanthroline doped in the FCS to develop a reddish coloration. The selective color development can be used to monitor ferrous ions in an FCS. The coloration obtained from a CMOS camera equipped with the microscope was quantified with RGB values obtained by image processing to estimate the amount and diffusion behavior of the dissolved 
ferrous ions. ${ }^{(27-29)}$ Herein, we validated the instrumentation by monitoring the dissolution behavior into FCSs formed in frozen solutions of $\mathrm{NaCl}$ and sucrose.

\section{Materials and Methods}

\subsection{Chemicals}

1,10-phenanthroline was purchased from Dojindo Laboratories (Japan). Iron(II) chloride, sodium chloride, sucrose, hydroxylamine sulfate, hydroxylamine chloride, and ammonium acetate were purchased from Kanto Chemical Co., Inc. (Japan). As the iron material to be investigated in the present work, pure iron wire $(\phi=1.0 \mathrm{~mm})$ was purchased from Nilaco Corp. (Japan). All the aqueous solutions were prepared with ultrapure water purified with a PURELAB Ultra Ionic system purchased from ELGA Labwater (United Kingdom). All the materials were used as received.

\subsection{Microscopic setup and image acquisition}

The setup for microscopic observation is schematically described in Fig. 1. A laboratorymade glass cell with a chamber height of $1 \mathrm{~mm}$ was placed on a Peltier unit (PU-50W, Takagi Manufacturing Corp., Japan) equipped with a stage of a microscope (BH2-FL, Olympus, Japan). The glass cell was fabricated by attaching two glass substrates to two glass spacers (thickness: 1 $\mathrm{mm}$ ) glued with epoxy resin as shown in Fig. 1. The glass substrates were made of borosilicate glass (S2441, Matsunami Glass Ind., Ltd., Japan). Although there is a possibility of contamination with metal ions from the borosilicate glass, the amount of contaminated ions is considered to be negligible since the solute concentration in the FCSs was sufficiently high. ${ }^{(30)}$ The bottom of the cell was covered with black paper to reduce the reflection of incident light at the Peltier surface, enabling nearly surface-specific observation of the ice film sample. The temperature of the Peltier unit was controlled with a Peltier controller (TDC-2020, Cell Systems, Japan). The cell was filled with an aqueous solution containing the solute ( $\mathrm{NaCl}$ or sucrose herein), 1,10-phenanthroline, and acetate-ammonium buffer ( $\mathrm{pH} 7.2$ ), and a sample wire was then placed in the chamber. The $\mathrm{pH}$ value of the solution to be frozen was 5.6, which was measured with a

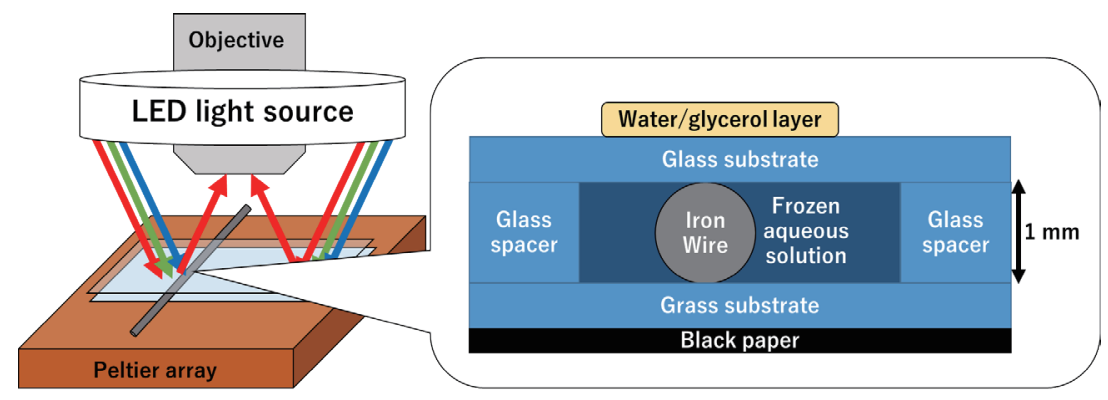

Fig. 1. (Color online) Schematic illustration of experimental setup for the diffusive-reflection microscopy. 
$\mathrm{pH}$ meter (LaQua, Horiba, Japan) prior to freezing. The iron wire was placed in the chamber immediately after wiping off anti-rust oil with a paper towel, after which a layer of a water/ glycerol mixture was deposited on the upper glass substrate to prevent dew formation. The solution was frozen by decreasing the temperature of the Peltier unit, and the change in color induced by the complexation of $\mathrm{Fe}(\mathrm{II})$ with 1,10-phenanthroline was observed. The elution behavior was monitored and diffusive-reflection microscopic images were captured with a CMOS camera (MU853B, AmScope). White light was irradiated to the sample with an LED light source (Model MIC-209, AmScope, USA) located above the sample. The acquired images were processed with ImageJ software (NIH, USA) to obtain RGB values. To simplify the quantification procedure, $\mathrm{R}$ values were used in the quantitative discussion on coloration.

\section{Results and Discussion}

Since the RGB value shows only the signal corresponding to the incident light to the CMOS sensors, it does not contain chemical information including concentration and chemical structure. Thus, the calibration curve to estimate the amount of ferrous ions was determined from the relationship between the R value and the concentration. Figure 2(a) shows microscopic images of the aqueous $\mathrm{FeCl}_{2}$ solution containing 1,10-phenanthroline with various concentrations of ferrous ions. The R values of the images in Fig. 2(a) are visualized in Fig. 2(b). In this study,

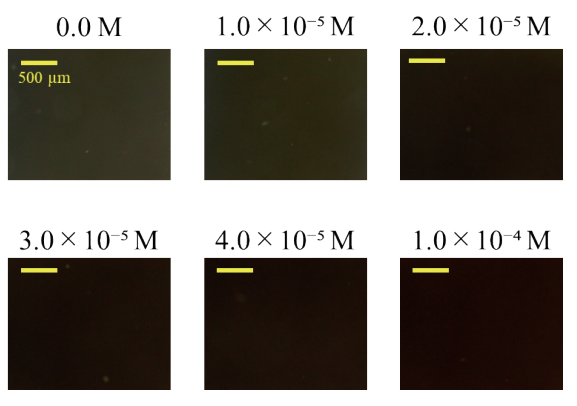

(a)

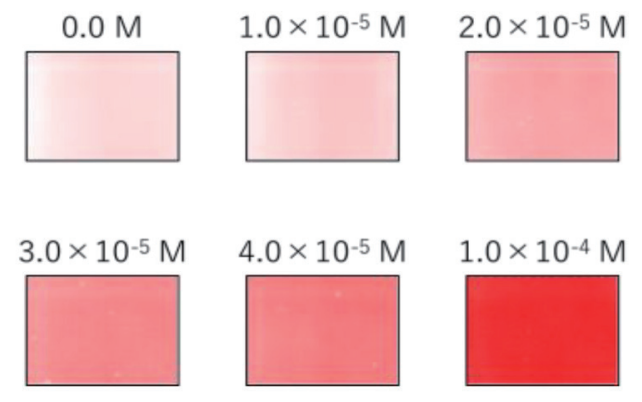

(b)

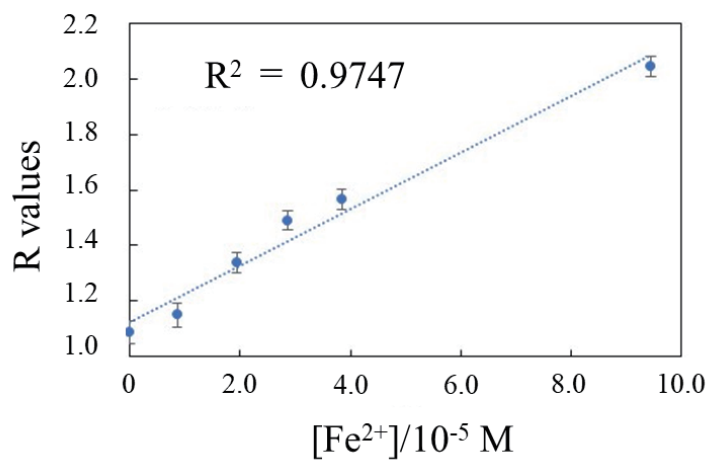

(c)

Fig. 2. (Color online) Diffusive-reflection microscopic images of Fe(II)-1,10-phenanthroline complex solutions in the measurement chamber. (a) Diffusive-reflection images of complex solutions with various concentrations of $\mathrm{FeCl}_{2}$; (b) maps of $\mathrm{R}$ values; (c) calibration curve determined from the microscopic images. 
we attempted to evaluate the coloration using the R values of the RGB over the given area of $35 \mu \mathrm{m} \times 35 \mu \mathrm{m}$. Although the intensity of the incident light was set to be weak for the surfaceselective observation, the $\mathrm{R}$ value was found to increase with the concentration of ferrous ions by image processing. Note that the $\mathrm{R}$ values were normalized with the luminance. The calibration curve determined from the results is shown in Fig. 2(c), which was used to obtain the following relationship between the $\mathrm{R}$ value and the concentration of ferrous ions:

$$
\mathrm{R} \text { value }=1.04 \times 10^{4}\left[\mathrm{Fe}^{2+}\right]+1.126 \text {, }
$$

where $\left[\mathrm{Fe}^{2+}\right]$ is the concentration of ferrous ions. The linearity of the calibration curve indicates that the $\mathrm{R}$ value is proportional to the concentration of $\mathrm{Fe}(\mathrm{II})-1,10$-phenanthroline complexes. The error bars in the curve reflect the unevenness of the color in each figure. Since the FCS is highly concentrated $\left(1.45 \mathrm{M} ; \mathrm{M} \equiv \mathrm{mol} \mathrm{L}^{-1}\right),{ }^{(31)}$ its refractive index is relatively high (1.350) and higher than that of water (1.337). ${ }^{(32)}$ The relative difference in the refractive indices is $1 \%$. Thus, the calibration curve was applied to our following experiment with frozen $\mathrm{NaCl}$ solutions. The following discussion on quantitative mapping is based on this calibration curve.

The coloration in the FCS in contact with a pure iron wire was then observed by diffusivereflection microscopy. Here, we describe the visualization process in detail. When an aqueous solution is frozen, phase separation occurs and solutes are expelled from the pure ice crystal to the FCS. The FCS thus contains the salt, 1,10-phenanthroline, hydroxylamine, and the buffer. When corrosion occurs at the iron/FCS interface, ferrous ions are dissolved in the FCS. Because the FCS contains hydroxylamine as a reductant, the amount of ferric ions is considered to be negligible. The dissolved ferrous ions are then complexed with 1,10-phenanthroline and the red coloration occurs. Note that there are some possible side reactions of ferrous ions with hydroxyl and acetate ions. For example, the stability constants for ferrous ions with acetate, mono, and $d i$ hydroxyl ions are $\sim 10^{2}, \sim 10^{5}$, and $\sim 10^{7}$, respectively, whereas that for Fe(II)-1,10-phenanthroline is $\sim 10^{21}$. (33) Thus, any side reactions are considered to be negligible in this system.

The dissolution behavior of ferrous ions was then studied with the established instrumentation. First, we characterized the morphology of the FCS formed in a frozen aqueous solution of $0.30 \mathrm{M} \mathrm{NaCl}$. Figure 3 shows a fluorescence microscopic image of the FCS. The solution

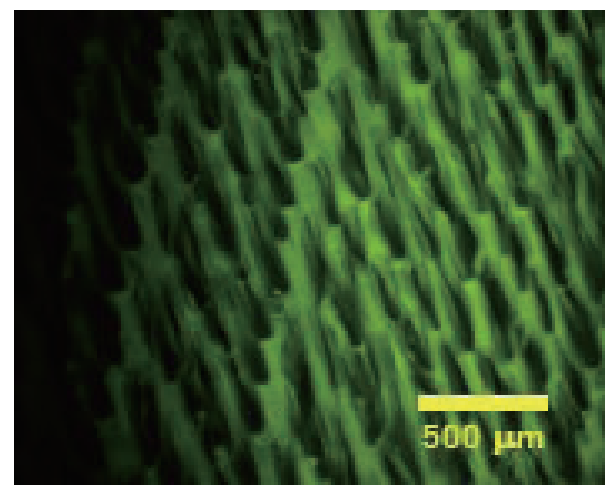

Fig. 3. (Color online) Fluorescence image of FCS formed in frozen $\mathrm{NaCl}$ solution $(0.30 \mathrm{M})$. The FCS was stained with fluorescein. 
contains fluorescein, allowing the FCS to be visualized with a fluorescence microscope. We found that the FCSs had channel-like structures with a width of several hundred micrometers and a length of several millimeters. It has been reported that the shape of the FCS depends on the solutes or the freezing temperature. ${ }^{(34)}$ In this study, the freezing and observation temperature was set to $-5.0^{\circ} \mathrm{C}$, which is higher than that in previous studies. This higher temperature leads to the slow crystallization of ice, resulting in the generation of large ice crystals and long FCS channels. Figure 4(a) shows diffusive-reflection microscopic images of the frozen aqueous $\mathrm{NaCl}$ in contact with the pure iron wire. The concentration of $\mathrm{NaCl}$ added as a solute was set to 0.30 M. Note that the black paper at the cell bottom absorbs light transmitted through the ice film, making the digital images dark but enabling specific observation of the sample surfaces. Since the refractive index of the FCS is different from that of ice, the ice/FCS interface can be observed as the border between black and yellowish parts in each image. The lighter part on the left side of each image is the pure iron wire and is due to light scattering at the wire. We observed the time dependences of the coloration of the FCS by analyzing the acquired images. To quantify the coloration, we obtained RGB values of the images with ImageJ software. The R values were then mapped on 2D planes as shown in Fig. 4(b). The color scale is also summarized in the figure. Note that the $\mathrm{R}$ values were normalized with the luminance. The iron wire/frozen aqueous solution interface can be observed as the boundary on the left side of each image, where the white and red parts are iron and the frozen solution, respectively. The coloration in the frozen solution regions was confirmed to become more intense over time.

The raw data of the R values in Fig. 4(b) reflect both the increased intensity of the coloration by the iron complexes and the intrinsic coloration of the FCS and ice, even though they are transparent. This indicates that the $\mathrm{R}$ value itself does not reflect the molar amount of ferrous ions dissolved from the iron wire. Thus, we calculated the differential of the $\mathrm{R}$ values $(\Delta \mathrm{R})$ to estimate the amount of Fe-1,10-phenanthroline complexes induced by the dissolution of ferrous ions from the iron wire. Figure 5(a) summarizes the $\Delta \mathrm{R}$ value at each time. $\Delta \mathrm{R}$ for each time was calculated by subtracting the $\mathrm{R}$ value at $0 \mathrm{~min}$ from that at a given time. The time herein is expressed by the differential value (e.g., $\Delta 10$ mins) since the $\Delta \mathrm{R}$ values are subtracted as mentioned above. We found that the R values in the FCS zone are higher than those in the ice

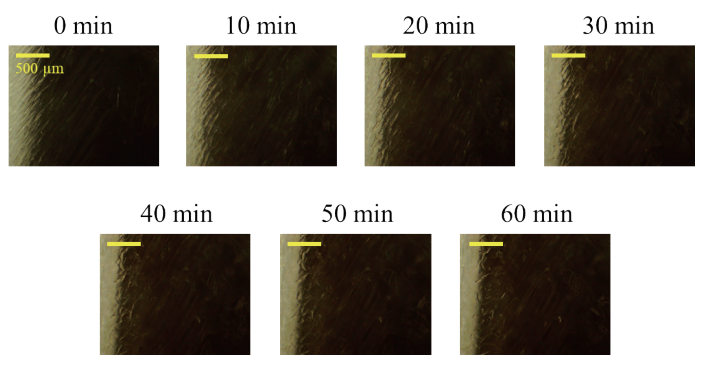

(a)

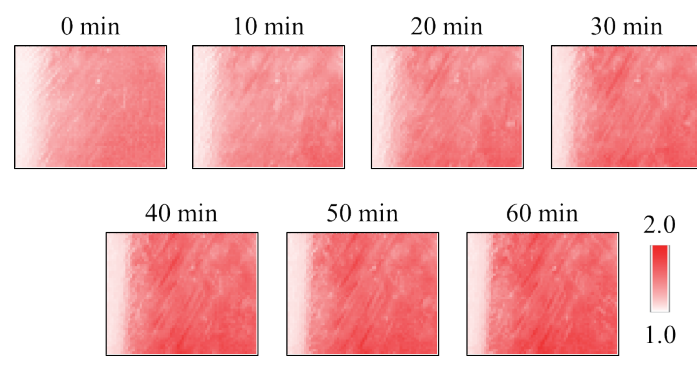

(b)

Fig. 4. (Color online) Diffusive-reflection microscopic images of frozen aqueous solution of $\mathrm{NaCl}(0.30 \mathrm{M})$ in contact with pure iron wire. (a) Diffusive-reflection images of the frozen solution after different times; (b) spatial maps of $R$ values in a given region of interest obtained by image processing with Image J software. 

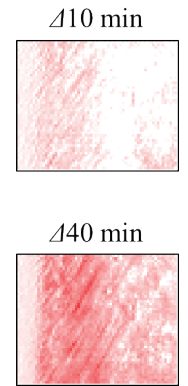
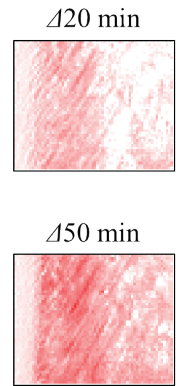

(a)
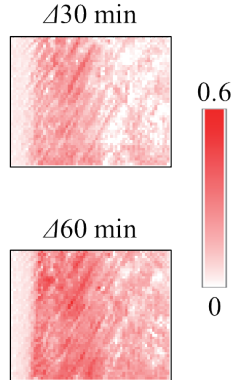

0

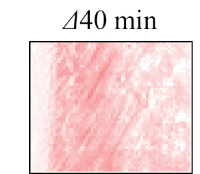

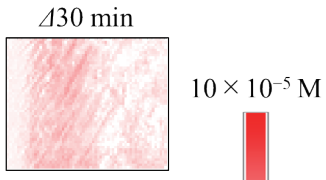

(b)

Fig. 5. (Color online) Spatial maps of the concentration of Fe(II)-1,10-phenanthroline complexes in the frozen aqueous $\mathrm{NaCl}(0.30 \mathrm{M})$ induced by the elution of ferrous ions from the pure iron wire. Spatial maps of (a) the $\Delta \mathrm{R}$ values and (b) concentrations calculated with Eq. (1).

crystal zones. The maps in Fig. 4 strongly reflect the shape of the FCS, which has a channel-like and network structure. Notably, $\Delta \mathrm{R}$ increased over time, and the area containing dissolved ferrous ions spread outward. Since Fig. 2(b) indicates that the R value reflects the amount of Fe(II)-1,10-phenanthroline complexes, the results confirm the dissolution and diffusion of the ferrous ions. Thus, the R value successfully illustrates the geometrical map of the FCS where the ferrous ions dissolved.

Figure 5(b) shows 2D maps of the concentration of Fe-1,10-phenanthroline complexes estimated from Fig. 4(a) and the calibration curves when the pure iron wire was placed in contact with $0.50 \mathrm{M}$ frozen aqueous $\mathrm{NaCl}$. The concentration of $\mathrm{Fe}(\mathrm{II})-1,10$-phenanthroline complexes in the FCS increased with time. Moreover, the concentration gradient in a single FCS channel was observed, and the concentration of ferrous ions was found to be higher near the iron wire and lower far from the wire. This 2D map indicates that the diffusion behavior of ferrous ions can be quantified by the present method. When transmittance microscopy is employed as an image acquisition system, the concentration estimated here can be affected by the overlaps of the FCS. This is because the transmitting light is absorbed by each FCS overlapping in the depth direction. The depth of an FCS was previously experimentally determined by X-ray fluorescence spectroscopy to be $\sim 100 \mu \mathrm{m}$. ${ }^{(35)}$ This indicates that the number of FCSs in the depth direction should be around 10 in the present measurement cell. However, diffusive-reflection microscopy with weak incident light focuses on the surface. Moreover, the black paper placed at the bottom of the cell absorbs the incident light transmitted through the sample, preventing the incident light from entering the sensing camera. Nevertheless, further morphological study of the FCS in the present measurement cell is necessary for a quantitative discussion of the above. It may be possible to reduce the thickness of the cell and the iron materials to $\sim 100 \mu \mathrm{m}$ to observe the elution behavior in a single FCS, which will be our future work.

To examine whether the present approach can be applied to frozen samples under various conditions, we observed the dissolution behavior of ferrous ions under two different conditions that affect the dissolution behavior: the initial concentration of the doped salt and the solute. Figure 6(a) shows the time dependence of the elution behavior with $0.50 \mathrm{M}$ frozen aqueous $\mathrm{NaCl}$ 


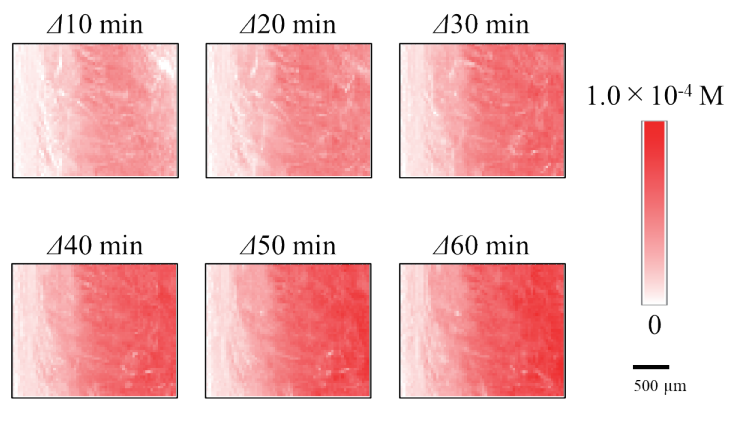

(a)

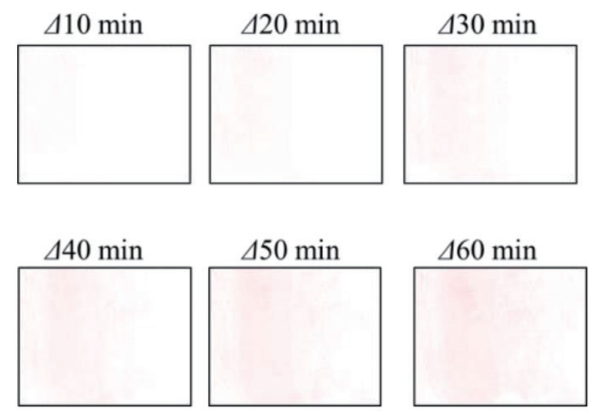

(b)

Fig. 6. (Color online) Spatial maps of the concentration of Fe(II)-1,10-phenanthroline complexes formed by the dissolution of ferrous ions from the pure iron wire in frozen aqueous solutions. (a) Frozen aqueous $\mathrm{NaCl}$ solution $(0.50 \mathrm{M})$; (b) frozen aqueous sucrose solution $(0.30 \mathrm{M})$.

solution. The colored area in Fig. 6(a) is larger than that in Fig. 4(b). This is attributed to thermodynamic equilibrium between ice and the FCS, which governs the constant solute concentration of the FCS at a given temperature. This indicates that the concentration of solutes in FCS is theoretically the same under each condition. More ferrous ions were dissolved from the iron wire when the frozen aqueous $\mathrm{NaCl}$ solution of $0.50 \mathrm{M}$ was placed in contact with the wire. This is attributed to the decrease in the cross-sectional area where iron comes in contact with the FCS. The higher the initial concentration of solutes is before freezing, the larger the volume of the FCS becomes. This indicates that the volumetric changes of the FCS strongly affect its crosssectional area. ${ }^{(36)}$ Thus, the larger the contact area, the greater the probability of the reaction of chloride ions coming in contact with the pure iron wire, leading to the increased dissolution of ferrous ions from the materials. Notably, the right part shows higher concentrations of Fe(II)-1,10-phenanthroline complexes than the part near the pure iron wire in Fig. 6(a). This is attributed to experimental errors induced by bubble formation in the ice film due to the light source $^{(37)}$ or the unstable morphology of the FCS due to the relatively high temperature of $-5.0^{\circ} \mathrm{C}$. These unfavorable phenomena are caused by the increase in the local temperature in the sample. Further improvement of the cooling system and control of the incident light intensity will help reduce these experimental errors. Nevertheless, the figure shows that the area near the iron wire gradually became reddish regardless of high values on the right side of each figure. Thus, we consider that mapping of dissolved ferrous ions near the iron wire was achieved and that further experimental improvements will enable the mapping of wider regions of interest for analysis. We also examined frozen aqueous solutions of sucrose as a non-ionic solute. Figure 6(b) summarizes the time dependence of the dissolved ferrous ion concentration along the direction of the FCS. Note that the calibration curve shown in Fig. 2(c) is not applicable to the frozen sucrose solution system due to the difference in the refractive index of the FCS $(n \approx 1.401)$. ${ }^{(38)}$ Thus, we determined the relationship between the $\mathrm{R}$ value and the iron concentration for the sucrose-concentrated system as

$$
\mathrm{R} \text { value }=9.42 \times 10^{3}\left[\mathrm{Fe}^{2+}\right]+1.49
$$


We found that the dissolution of ferrous ions tended to be greater for frozen $\mathrm{NaCl}$ solutions than for aqueous sucrose solutions. Since sucrose is not an ionic species, it is thus natural that sucrose-doped FCS has less ability to dissolve ferrous ions. The slight change in color in Fig. 6(b) indicates that trace amounts of ferrous ions were dissolved from the wire, possibly due to the presence of ionic species that can undergo complexation. The results herein exhibit the difference in the dissolution behavior between $\mathrm{NaCl}$ - and sucrose-doped ice, indicating that the existence of ionic species strongly promotes the dissolution of ferrous ions over nonvalent irons. To summarize the above discussion, the results obtained herein are consistent with those obtained by conventional studies on the corrosion of steel and iron materials, indicating that the present approach can be used for the in situ monitoring of ferrous ions dissolved from steel and iron materials to evaluate the corrosion behavior, assuming a low-temperature environment with the materials in contact with frozen aqueous solutions such as snow and sea ice. We believe that this methodology can also be applied to anti-corrosive steel materials covered with solid materials including polymers and gel-like materials to monitor the corrosion behavior microscopically, for which the conventional systems cannot easily perform in situ monitoring.

The FCS contains highly concentrated solutes because all the solutes are expelled from ice crystals to the liquid phase. In this study, the concentration of $\mathrm{NaCl}$ in the FCS was estimated to be $1.45 \mathrm{M}$. According to Foley's review, ${ }^{(19)}$ the corrosion mechanisms of steel materials differ in solutions with low and high salt concentrations. This indicates that further kinetic studies of the dissolution behavior in FCSs should be performed to elucidate the corrosion mechanism in FCSs. The present approach enables monitoring of both the change in concentration in a given area and the diffusion behavior of ferrous ions in FCS networks by simply taking microscopic images and carrying out image processing. Further analysis of the acquired images and maps would elucidate the effects of the concentration and species of ions on the corrosion mechanism in FCSs, which will be reported in the future.

\section{Conclusions}

We quantitatively visualized ferrous ions dissolved from pure iron wires into FCSs by a combination of transmittance microscopy and image analysis. When the FCS contains chloride ions, the dissolution reaction is accelerated compared with that of FCSs containing organic compounds. This indicates that the freeze-concentrated chloride ions in FCSs play an important role in the corrosion of iron materials. To the best of our knowledge, this is the first report of using the coloration of Fe(II)-1,10-phenanthroline complexes to visualize the chemical behavior of ferrous ions in an FCS surrounded by ice crystals, which is much simpler than conventional imaging techniques utilizing X-rays and a fluorescent probe. Improvement of the present method for quantitative analysis and mapping of the dissolved ferrous ions are now under way in our laboratory, which includes visualization of the dissolution behavior in single-layered FCS structures, cooling systems for freezing and temperature control, and a microscopic system for image acquisition with high evenness throughout the images. We believe that the simplicity of this approach will enable the screening and evaluation of the tolerance of steel materials to corrosion in low-temperature environments, including sea ice, polar climates, and lowtemperature instruments and machines. 


\section{Acknowledgments}

This work was supported by a Grant-in-Aid for Young Scientists (JP19K1559) and a Grant-in-Aid for Scientific Research on Innovative Areas (JP20H05203) from the Japan Society for the Promotion of Science. A. I. thanks Professor Tetsuo Okada (Department of Chemistry, Tokyo Institute of Technology, Japan) for valuable discussions on the elution behavior of ferrous ions. All authors thank the Advanced Instrumental Analysis Department of Utsunomiya University for their instrumental support.

\section{References}

1 N. Ahmad and A. G. MacDiarmid: Synth. Met. 78 (1996) 103. https://doi.org/10.1016/0379-6779(96)80109-3

2 D. W. DeBerry: J. Electrochem. Soc. 132 (1985) 1022. https://doi.org/10.1149/1.2114008

3 G. Mengoli, M. M. Musiani, B. Pelli, and E. Vecchi: J. Appl. Polym. Sci. 28 (1983) 1125. https://doi.org/10.1002/ app.1983.070280318

4 A. Bogdan, M.J. Molina, H. Tenhu, E. Mayer, and T. Loerting: Nat. Chem. 2 (2010) 197. https://doi.org/10.1038/ nchem.540

5 N. Takenaka, A. Ueda, and Y. Maeda: Nature 358 (1992) 736. https://doi.org/10.1038/358736a0

6 Y. Tasaki and T. Okada: J. Am. Chem. Soc. 134 (2012) 6128. http://pubs.acs.org/doi/abs/10.1021/ja301989d

7 H. Qu, M. Harada, and T. Okada: ChemElectroChem. 4 (2017) 35. https://doi.org/10.1002/celc.201600560

8 T. Muto, M. Harada, G. Fukuhara, and T. Okada: J. Phys. Chem. B 124 (2020) 3734. https://doi.org/10.1021/acs. jpcb.0c01451

9 A. Miyagawa, M. Harada, G. Fukuhara, and T. Okada: J. Phys. Chem. B 124 (2020) 2209. https://doi. org/10.1021/acs.jpcb.9b11345

10 K. Anzo, M. Harada, and T. Okada: J. Phys. Chem. A 117 (2013) 10619. https://doi.org/10.1021/jp409126p

11 A. Zelmer, N. Zhang, K. Komínková, D. Nachtigallová, H. H. Richnow, and P. Klán: Langmuir 31 (2015) 10743. https://doi.org/10.1021/acs.langmuir.5b02990

12 K. Iijima, M. Harada, G. Fukuhara, and T. Okada: J. Org. Chem. 85 (2020) 4525. https://doi.org/10.1021/acs. joc. $9 \mathrm{~b} 03415$

13 A. Inagawa, T. Ishikawa, T. Kusunoki, S. Ishizaka, M. Harada, T. Otsuka, and T. Okada: J. Phys. Chem. C 121 (2017) 12321. https://doi.org/10.1021/acs.jpcc.7b03792

14 A. Inagawa, M. Fukuyama, A. Hibara, M. Harada, and T. Okada: J. Colloid Interface Sci. 532 (2018) 231. https://doi.org/10.1016/j.jcis.2018.07.137

15 A. Inagawa, M. Harada, and T. Okada: J. Phys. Chem. C 123 (2019) 6062. https://doi.org/10.1021/acs. jpcc. 8 b12435

16 K. Tokumasu, M. Harada, and T. Okada: ChemPhysChem 18 (2017) 329. https://doi.org/10.1002/ cphc. 201601192

17 K. Kim, W. Choi, M. R. Hoffmann, H. I. Yoon, and B. K. Park: Environ. Sci. Technol. 44 (2010) 4142. https:// doi.org/10.1021/es9037808

18 S. P. M. Menacherry, K. Kim, W. Lee, C. H. Choi, and W. Choi: Environ. Sci. Technol. 52 (2018) 13766. https:// doi.org/10.1021/acs.est.8b04484

19 R. T. Foley: Corrosion 26 (1970) 58. https://doi.org/10.5006/0010-9312-26.2.58

20 M. Doi, M. Harada, and T. Okada: ACS Earth Space Chem. 5 (2021) 1544. https://doi.org/10.1021/ acsearthspacechem.1c00071

21 H. Qu, M. Harada, and T. Okada: ChemElectroChem 4 (2017) 35. https://doi.org/10.1002/celc.201600560

22 T. Hashimoto, Y. Tasaki, M. Harada, and T. Okada: Anal. Chem. 83 (2011) 3950. http://pubs.acs.org/doi/ abs/10.1021/ac200785n

23 T. Hashimoto, M. Harada, S. Nojima, and T. Okada: ChemPhysChem 14 (2013) 3410. https://doi.org/10.1002/ cphc. 201300380

24 J. S. Kim and K. Kim: Chem. Commun. 55 (2019) 12136. https://doi.org/10.1039/c9cc05809e

25 G. Køhler, S. Solar, and N. Getoff: J. Phys. Sci. A 35 (1980) 1201. https://doi.org/10.1515/zna-1980-1113

26 M. H. Noiré and B. Duréault: Sens. Actuators, B 29 (1995) 386. https://doi.org/10.1016/0925-4005(95)01712-7

27 A. Inagawa, A. Sasaki, and N. Uehara: Talanta 216 (2020) 120952. https://doi.org/10.1016/j.talanta.2020.120952 
28 A. Inagawa, K. Saito, A. Sasaki, and N. Uehara: Data Br. 31 (2020) 105998. https://doi.org/10.1016/j. talanta.2020.120952

29 A. Inagawa and N. Uehara: Bunseki Kagaku 69 (2020) 693. https://doi.org/10.2116/bunsekikagaku.21.1551

30 A. Mizuike and A. Iino: Anal. Chim. Acta 124 (1981) 427. https://doi.org/10.1016/S0003-2670(01)93592-5

31 Y. Tasaki and T. Okada: Anal. Chem. 83 (2011) 9593. https://doi.org/10.1021/ac202378m

32 K. M. Aly and E. Esmail: Opt. Mater. 2 (1993) 195. https://doi.org/10.1016/0925-3467(93)90013-Q

33 The Chemical Society of Japan: Chemical Index 4th Edition (Maruzen, Tokyo, 1993) p. II-325.

34 M. Uyama, M. Harada, T. Tsukahara, and T. Okada: J. Phys. Chem. C 117 (2013) 24873. https://doi.org/10.1021/ jp408722x

35 K. Tokumasu, M. Harada, and T. Okada: Langmuir 32 (2016) 527. https://doi.org/10.1021/acs.langmuir.5b04411

36 A. Inagawa, M. Harada, and T. Okada: Sci. Rep. 5 (2015) 17308. https://doi.org/10.1038/srep17308

37 S. Mae: J. Glaciol. 17 (1976) 111. https://doi.org/10.3189/S0022143000030768

38 D. F. Charles: Anal. Chem. 37 (1965) 405. https://doi.org/10.1021/ac60222a027

\section{About the Authors}

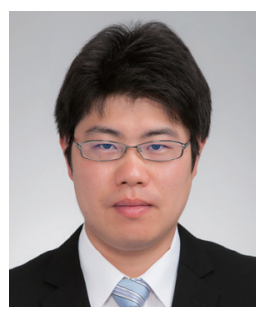

Arinori Inagawa received his B.S. (2013), M.S. (2015), and Ph.D. (2018) degrees in analytical chemistry from Tokyo Institute of Technology, Japan. After his postdoctoral research at Tokyo Institute of Technology, he was appointed as an assistant professor at Utsunomiya University, Japan, in 2018. His research interests are separation science, phase separation, microfluidics, and microspectroscopy for interfacial analysis.

(ainagawa@cc.utsunomiya-u.ac.jp)

Minami Maeda received her B.Eng. degree from Utsunomiya University, Japan, in 2021 and currently is a master's course student at the Graduate School of Utsunomiya University. Her research interest is the observation and evaluation of corrosion phenomena of steel materials in polar and cryogenic environments.

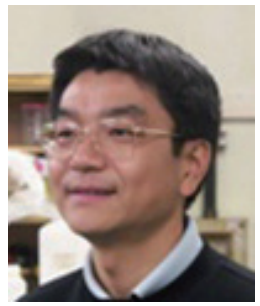

Nobuo Uehara received his B.S. (1986), M.S. (1988), and Ph.D. (1996) degrees from Tohoku University, Japan. He was appointed as an assistant professor at Utsunomiya University, Japan, in 1988. He is currently a full professor at Utsunomiya University. His fields of interest are the fabrication of functional materials composed of stimuli-responsive polymers and metal nanoparticles and their application to analytical chemistry. (ueharan@cc.utsunomiya-u.ac.jp) 